\title{
The Renaissance of Microbiology: The Necessary Future for Matrix Assisted Laser Desorption Ionisation Mass Spectrometry Based Bio Typing \\ Matthew B O'Rourke ${ }^{1 *}$, Steven P Djordjevic ${ }^{2}$ and Matthew P Padula ${ }^{1}$
}

${ }^{1}$ Proteomics Core Facility, University of Technology Sydney, Cnr Harris and Thomas St, Ultimo, NSW, 2007 Australia

${ }^{2}$ The iThree Institute, University of Technology Sydney, Cnr Harris and Thomas St, Ultimo, NSW, 2007 Australia

Keywords: MALDI; Biotyping; Bacterial identification; Mass spectrometry

\section{Introduction}

Diagnostic microbiology and the traditional biochemical and the phenotype based morphological techniques that accompany it, have been a staple of pathological testing for the last 80 years [1]. Current methods for elucidating the agent responsible for causing an infection in humans rely on the observation of bacterial cells as well as biochemical testing aimed at utilising the metabolism of the organism as an indicator of its identity [2]. Following these observations, an experienced pathologist will then report on the likely identity of the pathogen leading to the treatment of a patient with appropriate antibiotics. Systems such as the API 20E (Biomerieux) have made performing these tests a much more streamlined and standardised procedure, however the bacterial identities are not always correct or specific enough to be of diagnostic use, only offering genus or species level identifications [3]. Biochemical based tests are also often expensive and require incubation periods that lengthen the time required for diagnosis thereby increasing the morbidity of patients with serious infections [4]. When all the above is considered, there was a drastic need for improvement in microbiological diagnoses which arrived in the form of Biotyping, first pioneered by Daltonics [5].

Biotyping is an analysis technique that uses Matrix Assisted Laser Desorption Ionisation Mass Spectrometry (MALDI-MS) to identify microorganisms by analysing the molecules that are in the highest abundance, such as ribosomal proteins and cold-shock proteins, and are the most easily ionisable from a range of clinical samples. In short, a small amount of bacterial cells are grown on appropriate agar media (or directly sampled by colony picking) and 1-2 colonies are collected and prepared according to a proprietary protocol to extract molecules that can be analysed by the mass spectrometer [6]. The instrument then analyses the sample and compares the data back to a reference database that contains a spectral library of known organisms. Any matching entries are then reported with a simplified scoring system that indicates the confidence in the identification; red for not confident, yellow for partially confident and green for very confident [5]. Clinicians are then provided with the report and the appropriate action is taken to treat the patient; The total process from human sample to identification takes $\sim 25 \mathrm{~h}$ depending on the length of time needed to grow the sample on agar media with sample preparation taking only $20 \mathrm{~min}$. There are numerous advantages to such a system including faster responses and therefore faster treatment for patients, reduced costs, automation of sample analysis and a simple protocol that can be performed by "walk up" users. From a cost perspective individual samples have been determined as costing $€ 1.43$ per sample as opposed to $\sim € 10-13$ for systems such as the API 20E biochemical test strip. When combined with gram staining and other routine phenotypic tests costs can easily be $\sim € 25$ per sample [7]. However, despite these benefits, Biotyping is still in need of significant improvement before it can truly replace traditional methodologies.
In recent years we have begun our own investigation into the application of MALDI for bacterial diagnosis and, by applying our extensive knowledge in MALDI-TOF based technology [8,9], have discerned a number of key limitations with currently implemented Biotyping methodologies. The greatest limitation is that several major pathogen groups, responsible for a large proportion of clinical infections, such as Staphylococcus spp., Streptococcus spp. and Escherischia coli/ Shigella subgenus, are indistinguishable beyond the genus level [10] meaning they cannot be accurately identified without further testing. In addition to this, there is a high rate of misidentification [11] with a range $~ 85 \%-97 \%$ of organisms able to be accurately identified to genus or species level, depending on the organism. It should be noted that the identification to strain level has not yet been reliably achieved and is still in its developmental infancy [12].

Another major issue is that current methodologies do not ensure the death of the pathogen before loading into the instrument for analysis. We have personally repeated the available methodologies and have confirmed this to be true; we will be publishing our findings shortly (manuscript in preparation). This poses a risk of infection by potentially high risk group human pathogens to pathology staff as well as engineers who are responsible for servicing the instrument.

As stated above, the rate of misidentification and the level to which organisms are identified are in need of significant improvement. However in the opinion of these authors, certain additional directions need to be explored to fully realise the potential of this technology.

Currently no single microbiological diagnostic in existence is able to accurately discern the identity of a pathogen from a clinical sample to strain or sub-strain accuracy, meaning that degrees of pathogenicity, that can often only be determined at strain level, are not able to be discerned. A good example of this is genus/species $E$. coli which contains a range of pathogenic and non-pathogenic strains. These including BL21 cells, used for recombinant protein production workflows [13] and O104:H4 Enteroaggregative -haemorrhagic strains that cause severe disease [14], but current Biotyping methodologies are unable to distinguish between a benign and pathogenic strain. If Biotyping were improved in the ways mentioned above and implemented in hospitals and pathology laboratories, the quality of patient care will dramatically increase and the current cost of healthcare will decrease as the detection

*Corresponding author: Matthew B O'Rourke, Proteomics Core Facility, University of Technology Sydney, Cnr Harris and Thomas St, Ultimo, NSW, 2007 Australia, Tel: +61295148374; E-mail: matthew.padula@uts.edu.au

Received July 05, 2016; Accepted July 31, 2016; Published August 08, 2016

Citation: O'Rourke MB, Djordjevic SP, Padula MP (2016) The Renaissance of Microbiology: The Necessary Future for Matrix Assisted Laser Desorption Ionisation Mass Spectrometry Based Bio Typing. J Microb Biochem Technol 8: 373-374. doi: 10.4172/1948-5948.1000311

Copyright: (c) 2016 O'Rourke MB, et al. This is an open-access article distributed under the terms of the Creative Commons Attribution License, which permits unrestricted use, distribution, and reproduction in any medium, provided the original author and source are credited. 
Citation: O'Rourke MB, Djordjevic SP, Padula MP (2016) The Renaissance of Microbiology: The Necessary Future for Matrix Assisted Laser Desorption Ionisation Mass Spectrometry Based Bio Typing. J Microb Biochem Technol 8: 373-374. doi: 10.4172/1948-5948.1000311

and identification of pathogens will become faster and more accurate leading to faster more targeted treatments.

It is extensively documented in the literature that antibiotic resistance is rapidly spreading and that bacteria are able to rapidly transfer this resistance to sensitive cells through mechanisms such as lateral gene transfer [14]. There is currently no rapid assay available to show the presence or specificity of antibiotic resistance in microorganisms. Antibiotic resistance and the resulting emergent diseases have a significant impact on global health systems with certain virulent pathogens such as extreme resistance Pseudomonas aeruginosa requiring targeted treatment with potentially toxic $4^{\text {th }}$ line antibiotics [15]. If a rapid diagnostic could be applied to determine the antibiotic resistance of the bacteria infecting a patient without determination of the bacteria's identity, then treatments could become more targeted leading to increased patient outcomes and a reduction in emerging pathogens resulting from treatments with broad spectrum antibiotics [16].

MALDI Biotyping is a technique that is currently showing great potential in the field of microbial identification. With further exploration and expansion of this technique it could well prove to be the next ubiquitous diagnostic platform for human clinical samples. However, current implementations of this technology are in need of significant improvement in the areas of antibiotic resistance, identification accuracy and taxonomic level of identification. Once these areas have been addressed, microbial diagnostics will finally see a true renaissance.

\section{References}

1. Burke $V(1922)$ Notes on the gram stain with description of a new method. $J$ Bacteriol 7: 159-182.

2. Morgulis S, Levine VE (1920) Decomposition of hydrogen peroxide by organic compounds and its Bearing On The Catalase Reaction. Science 52: 202-204.

3. Butler DA, Lobregat CM, Gavan TL (1975) Reproducibility of the analytab (API 20E) system. J Clin Microbiol 2: 322-326.
4. Roca I (2015) The global threat of antimicrobial resistance: science for intervention. New Microbes and New Infections 6: 22.

5. Daltonics B (2008) Microorganism identification and classification based on MALDI-TOF MS fingerprinting with MALDI Biotyper Application Note \#MT-80.

6. Drancourt M (2010) Detection of microorganisms in blood specimens using matrix-assisted laser desorption ionization time-of-flight mass spectrometry: A review. Clin Microbiol Infect 16: 1620.

7. Seng P, Drancourt M, Gouriet F, La Scola B, Fournier PE, et al. (2009) Ongoing revolution in bacteriology: Routine identification of bacteria by matrixassisted laser desorption ionization time-of-flight mass spectrometry. Clin Infect Dis 49: 543-551.

8. O'Rourke MB, Djordjevic SP, Padula MP (2015) A non-instrument-based method for the analysis of formalin-fixed paraffin-embedded human spinal cord via matrix-assisted laser desorption/ionisation imaging mass spectrometry. Rapid Communications in Mass Spectrometry 29: 1836.

9. O'Rourke MB, Raymond BBA (2015) Djordjevic SP and Padula MP, A versatile cost-effective method for the analysis of fresh frozen tissue sections via matrix-assisted laser desorption/ionisation imaging mass spectrometry. Rapid Communications in Mass Spectrometry 29: 637.

10. Sogawa K, Watanabe M, Sato K, Segawa S, Ishii C, et al. (2011) Use of the MALDI BioTyper system with MALDI-TOF mass spectrometry for rapid identification of microorganisms. Anal Bioanal Chem 400: 1905-1911.

11. Patel R (2015) MALDI-TOF MS for the diagnosis of infectious diseases. Clin Chem 61: 100-111.

12. Calderaro A, Piergianni M, Montecchini S, Buttrini M, Piccolo G, et al. (2016) MALDI-TOF mass spectrometry as a potential tool for Trichomonas vaginalis identification. BMC Infect Dis 16: 261

13. Yu Z (2016) High level extracellular production of a recombinant alkaline catalase in E. coli BL21 under ethanol stress and its application in hydrogen peroxide removal after cotton fabrics bleaching. Bioresour Technol 21: 303.

14. Wyrsch, Ethan R (2016) "Genomic Microbial Epidemiology Is Needed to Comprehend the Global Problem of Antibiotic Resistance and to Improve Pathogen Diagnosis." Frontiers in Microbiology 7: 843. PMC. Web.

15. Liew YX, Tan TT, Lee W, Ng JL, Chia DQ, et al. (2013) Risk factors for extremedrug resistant $P$ seudomonas aeruginosa infections in patients with hematologic malignancies. Am J Infect Control 41: 140-144.

16. Ryzhov V, Fenselau C (2001) Characterization of the protein subset desorbed by MALDI from whole bacterial cells. Anal Chem 73: 746-750. 